\title{
Forskolin attenuates retinal inflammation in diabetic mice
}

\author{
ZHI-PENG YOU*, BIN XIONG* ${ }^{*}$ YU-LAN ZHANG, LU SHI and KE SHI \\ Department of Ophthalmology, The Second Affiliated Hospital, Nanchang University, \\ Nanchang, Jiangxi 330006, P.R. China
}

Received April 30, 2017; Accepted November 14, 2017

DOI: $10.3892 / \mathrm{mmr} .2017 .8106$

\begin{abstract}
The present study aimed to investigate the effect of forskolin on retinal inflammation under diabetic conditions. C57BL/6 mice were randomly divided into normal control, diabetic control and forskolin treatment groups. The diabetic model was established by intraperitoneal injection of streptozotocin. The forskolin treatment group received intragastrical administration of forskolin for 12 weeks, the other two groups received an equal amount of PBS. At 21 weeks following diabetic induction, an immunoblotting test was conducted to investigate the expression of two inflammatory factors: Intercellular adhesion molecule-1 (ICAM-1) and tumor necrosis factor- $\alpha$ (TNF- $\alpha)$. Glucose concentration was additionally calculated. A leukostasis assay was utilized to compare microvasculature pathological alterations. It was demonstrated that retinal glucose concentration of diabetic control and forskolin treatment were both increased compared with normal control, however the forskolin treatment group was only $\sim 68.06 \%$ of the diabetic control due to downregulated glucose transporter 1 expression. The expression of ICAM-1 and TNF- $\alpha$ were upregulated in the forskolin treatment and diabetic control groups compared with the normal control, however these two inflammatory factor expression levels in the forskolin treatment group were $\sim 68.75$ and $75.37 \%$ of diabetic control. It was additionally observed that there were less adherent leukocytes in retinal microvasculature in the forskolin treatment group compared with diabetic control. All the differences observed were significant. Overall, by means of limiting glucose transport into the retina via forskolin, the retinal environment with lower glucose concentration alleviates the inflammatory response under diabetic conditions.
\end{abstract}

Correspondence to: Dr Ke Shi, Department of Ophthalmology, The Second Affiliated Hospital, Nanchang University, 1 Minde Road, Nanchang, Jiangxi 330006, P.R. China

E-mail: 86325294@qq.com

*Contributed equally

Key words: glucose transporter type 1, forskolin, diabetic retinopathy, leukostasis, inflammation marker

\section{Introduction}

Diabetic retinopathy (DR) is a major blinding eye disease and often causes vision loss or even blindness due to macular oedema, retinal detachment and vitreous haemorrhage. It has been predicted that the number of patients with diabetes would increase to 642 million in 2040 (1). Studies showed that under a high glucose environment, excessive oxidative stress products in the retina (2), activation of the renin-angiotensin system (3), and an increase in advanced glycation end products (4) initiated damage in retinal tissue cells, thus causing a series of subsequent pathological changes.

Inflammation is an important process in the microangiopathy of DR and many inflammatory mediators are activated in the pathogenesis (5). Previous studies have reported that inflammation could induce retinal biochemical and molecular changes, leading to vision-threaten complications such as diabetic macular edema (DME) (6). Corticosteroids was effective in the treatment of DME proved that inflammation response plays a vital role in DR (7). However the present study merely focused on the upstream of inflammation in DR, hyperglycemia.

Glucose in the retina is transported from blood circulation. Glucose uptake by retinal tissue cells depends on one type of glucose-transporting carrier protein family, glucose transporters (GLUTs). GLUT1 is an important member of the GLUT family and is the only transporter that allows glucose to pass through the blood-retinal barrier (8). Forskolin is a type of diterpenoid derivative extracted from Coleus forskohlii. Forskolin can inhibit the glucose transport by GLUT1 (9). Our group aimed to use forskolin to inhibit the GLUT1 transport of glucose into the retina and thus reduce the glucose level in retinal tissues; consequently, we could determine whether inhibition of GLUT1 could control the glucose level in retinal tissues and alleviate inflammation response of DR.

\section{Materials and methods}

Reagents and instruments. Streptozotocin (STZ), forskolin, and the glucose content detection reagent kit were all from Sigma-Aldrich (Merck KGaA, St. Louis, MO, USA). The protein quantitation reagent kit was from Bio-Rad Laboratories, Inc. (Hercules, CA, USA). Fluorescein isothiocyanate conjugated concanavalin A (FITC-ConA) was from Vector Laboratories (Burlingame, CA, USA). Rabbit anti-mouse 
GLUT-1, intracellular cell adhesion molecule-1 (ICAM-1) and tumour necrosis factor- $\alpha$ (TNF- $\alpha$ ) primary antibodies and goat anti-rabbit secondary antibody were all from Millipore Corporation (St. Charles, MO, USA). Citric acid buffer was from Tiangen Biotech (Beijing, China). The electronic analytic balance was from Jingke Balance Factory (Shanghai, China). The spectrophotometer was from Spectro (Kleve, Germany). The inverted fluorescence microscope was from Olympus Corporation (Tokyo, Japan).

Experimental animals and grouping. This study was complied with the Animal Research: Reporting of In Vivo Experiments (ARRIVE) guidelines and carried out in strict accordance with the recommendations in the Guide for the Care and Use of Laboratory Animals of the National Institutes of Health (NIH Publications no. 8023, revised 1978). The protocol was approved by the Committee on the Ethics of Animal Experiments of Nanchang University. A total of 27 healthy, inbred, 8-week-old, male, and clean-grade C57BL/6 mice without eye diseases and with a body weight of 20-30 g were purchased from the Department of Animal Sciences of Nanchang University. The mice were numbered using ear tags and randomly divided into a normal control group, diabetic control group, and forskolin treatment group. Each group had 9 animals. For establishment of the DM model, mice were fasted for $8 \mathrm{~h}$ and then received an intraperitoneal injection of STZ for 5 consecutive days. Before injection, STZ was dissolved in $\mathrm{pH} 4.5,0.01 \mathrm{M}$ citric acid buffer. The diabetic control group and the forskolin treatment group received STZ at a dose of $55 \mathrm{mg} \cdot \mathrm{kg}^{-1}(10)$, and the normal control group received an intraperitoneal injection of an equal amount of citric acid buffer. Tail vein blood samples were collected on day 7 to measure blood glucose. The model was successfully established when the blood glucose level was greater than $300 \mathrm{mg} \cdot \mathrm{dl}^{-1}$. After model establishment, the forskolin treatment group received intragastric administration of forskolin at $50 \mathrm{mg} \cdot \mathrm{kg}^{-1}$ week ${ }^{-1}$ for 12 consecutive weeks, and the normal control group and the diabetic control group received an equal amount of PBS.

Detection of glucose levels in retinal tissues. Six mice from each of the 3 groups were sacrificed by spinal dislocation, and 6 left eyeballs were removed to measure the glucose levels in the retina. The right eyeballs were used for subsequent western blot analysis. Retinal tissues were collected, added to $50 \mu \mathrm{l}$ deionized water, and heated to $70-75^{\circ} \mathrm{C}$ for $15 \mathrm{~min}$. The samples were then sonicated for $30 \mathrm{sec}$ and centrifuged for $20 \mathrm{~min}$. A total of $35 \mu \mathrm{l}$ supernatant was added to $165 \mu \mathrm{l}$ glucose concentration assay reagent, and the standard curve and the blank control were set up. The absorbance of the samples was measured using a spectrophotometer, and the glucose concentration was calculated using SPECTROstar Nano MARS software. Next, $10 \mu \mathrm{l}$ supernatant was added to $190 \mu \mathrm{l}$ protein concentration determination reagent, and the standard curve and a blank control were set up. The absorbance of the samples was measured using a spectrophotometer, and the protein concentration was calculated using SPECTROstar Nano MARS software. The retinal glucose level was expressed as nmol glucose $\cdot m g$ protein ${ }^{-1}$, and the calculation formula was $\mathrm{GxGV} \cdot \mathrm{GMW}^{-1} \cdot(\mathrm{PxPV})^{-1}$, with the following parameters:
$\mathrm{G}$, glucose concentration $\left(\mathrm{ng} \cdot \mathrm{ml}^{-1}\right)$; $\mathrm{GV}$, volume of glucose content determination reaction solution $(\mathrm{ml})$; $\mathrm{P}$, protein concentration $\left(\mathrm{mg} \cdot \mathrm{ml}^{-1}\right)$; $\mathrm{PV}$, volume of protein concentration determination reaction solution $(\mathrm{ml})$; and GMW, molecular weight of glucose (180.2).

Western blot analysis. The retinal tissues of the above 6 removed right eyeballs of the mice in the 3 groups were collected and placed in Eppendorf tubes with $200 \mu 1$ lysis buffer. Samples were sonicated for $30 \mathrm{sec}$ and centrifuged for $20 \mathrm{~min}$. The supernatant was collected, and the protein concentrations of the samples were determined. Equal amounts of protein samples were collected and subjected to SDS-PAGE electrophoresis. Proteins were transferred onto a membrane for 90-120 min. The membrane was incubated with the GLUT-1, ICAM-1 and TNF- $\alpha$ primary antibodies at $75^{\circ} \mathrm{C}$ overnight. The next day, the membrane was washed 3 times and incubated with the secondary antibody at room temperature for $1 \mathrm{~h}$. The developing solution was then added in a dark room for gel image analysis; in addition, the grey scale values of the protein bands were measured.

Leukostasis assay in retinal blood vessels. Three mice from each of the 3 groups were anaesthetized using a mixed solution of ketamine $\left(10 \mathrm{mg} \cdot \mathrm{kg}^{-1}\right)$ and xylazine $\left(60 \mathrm{mg} \cdot \mathrm{kg}^{-1}\right)$. The thoracic skin and ribs of the mice were cut to expose the thoracic cavity. The descending aorta was closed by clamping, the right atrial appendage was cut, and a $27 \mathrm{G}$ needle was inserted into the left ventricle. A mixture of $10 \mathrm{ml}$ PBS and heparin $\left(0.1 \mathrm{mg} \cdot \mathrm{ml}^{-1}\right)$ was first perfused to wash out non-adherent leukocytes. Next, adherent leukocytes were labelled with a mixed solution containing $20 \mu \mathrm{g} \cdot \mathrm{ml}^{-1} \mathrm{PBS}$ and FITC-ConA (5 mg.kg-1). FITC-ConA that did not bind to leukocytes was washed out using $10 \mathrm{ml}$ PBS. After the mice were sacrificed, 6 eyeballs were removed from each group and directly placed in $4 \%$ paraformaldehyde for $1 \mathrm{~h}$. Next, the retinal tissues were flat mounted and counted under a fluorescence microscope.

Statistical analysis. Statistical analyses were performed using SPSS 17.0 (SPSS, Inc., Chicago, IL, USA) statistical software. The body weight, glucose levels, retinal glucose levels, and grey scale density results of western blot were expressed as mean \pm SD. A comparison between groups was performed using one-way analysis of variance (ANOVA) test with post hoc contrasts by Student-Newman-Keuls test. $\mathrm{P}<0.05$ was considered to indicate a statistically significant difference.

\section{Results}

Establishment of the diabetic model and measurement of body weight and blood glucose levels in the three groups. All blood glucose levels of the 27 males C57BL/6 mice (diabetic control and forskolin treatment groups) used for diabetes induction were higher than $300 \mathrm{mg} / \mathrm{dl}$ at 7 day after streptozotocin intraperitoneal injection. We measured the body weight and blood glucose levels of the mice at 21 weeks after diabetes induction. The results showed that there were no significant differences in the body weight of the mice among 
Table I. Body weight and blood glucose levels of the three groups.

\begin{tabular}{lcccccc}
\hline & \multicolumn{2}{c}{ Body weight $(\mathrm{g})$} & & \multicolumn{2}{c}{ Blood glucose level (mg/dl) } \\
\cline { 2 - 3 } \cline { 5 - 6 } Group & $\begin{array}{c}\text { At 1 day after the } \\
\text { diabetes induction }\end{array}$ & $\begin{array}{c}\text { At 21 weeks after the } \\
\text { diabetes induction }\end{array}$ & & $\begin{array}{c}\text { At 1 day after the } \\
\text { diabetes induction }\end{array}$ & $\begin{array}{c}\text { At 21 weeks after the } \\
\text { diabetes induction }\end{array}$ \\
\hline Normal control & $26.32 \pm 2.25$ & $36.31 \pm 2.98$ & & $178.41 \pm 19.58$ & $169.53 \pm 26.25$ \\
Diabetic control & $25.83 \pm 2.00$ & $25.36 \pm 3.02^{\mathrm{a}}$ & & $343.36 \pm 61.52$ & $378.51 \pm 51.25^{\mathrm{a}}$ \\
Forskolin treatment & $26.12 \pm 1.98$ & $26.33 \pm 3.16^{\mathrm{a}}$ & & $348.15 \pm 63.53$ & $363.52 \pm 60.14^{\mathrm{a}}$ \\
\hline
\end{tabular}

aP $<0.01$ vs. normal control $n=9$, mean \pm SD.
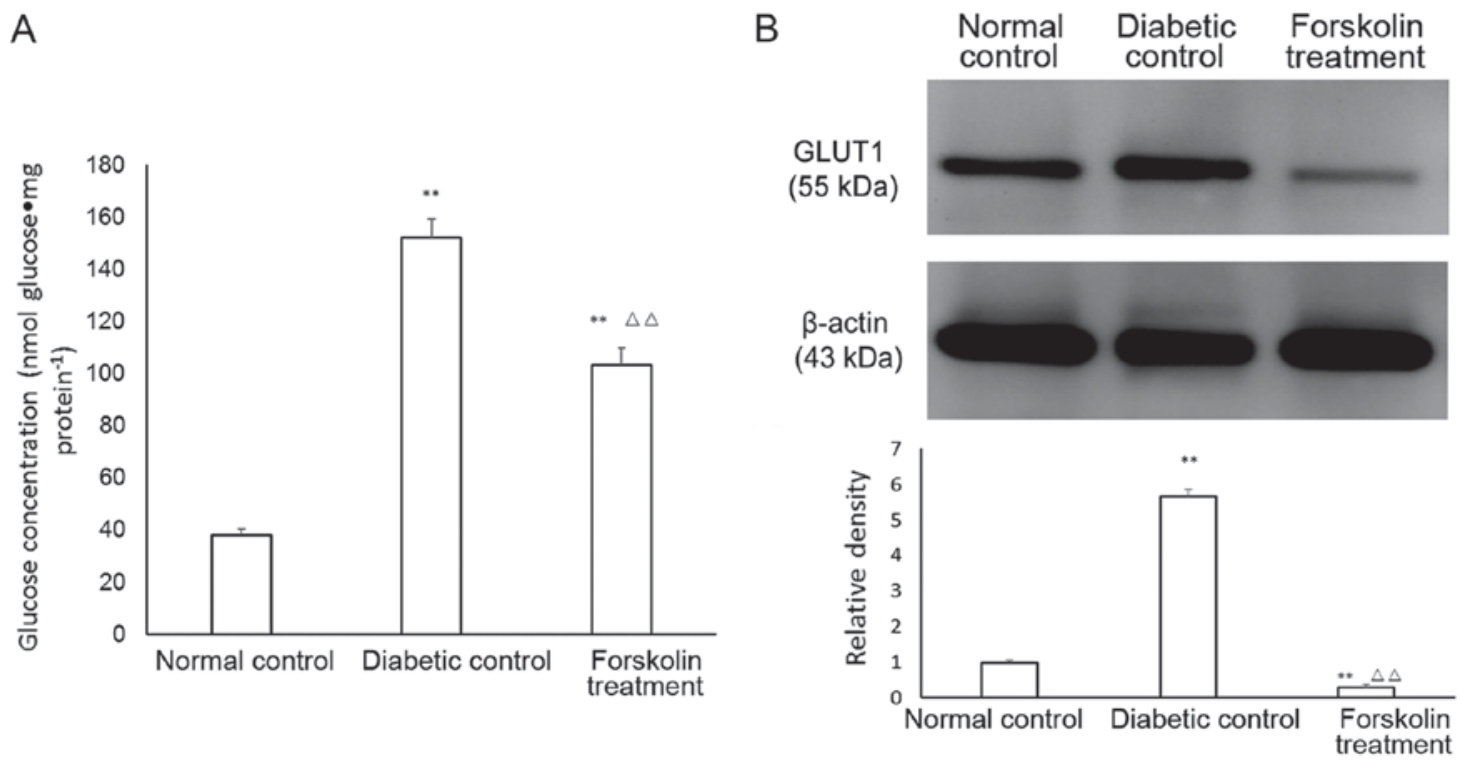

Figure 1. Retinal glucose concentration and GLUT1 expression of three groups mice. (A) Retinal glucose concentration of three groups mice; (B) GLUT1 expression in the retina of three groups. Data are representative of three independent experiments and values are expressed in mean $\pm \mathrm{SD}$. ${ }^{* *} \mathrm{P}<0.01 \mathrm{vs}$. normal control; ${ }^{\Delta \Delta} \mathrm{P}<0.01$ vs. diabetic control. $\mathrm{n}=6$.

the three groups when the diabetes was successfully induced. At 21 weeks after diabetes induction, the body weight of the normal control group was significantly higher than that of the diabetic control and forskolin treatment groups by 43.18 and $37.90 \%$, respectively.

We also measured the blood glucose levels at 1 day and 21 weeks after the diabetes induction. The blood glucose levels of the normal control group were lower than those of the diabetic control group by 48.04 and $55.21 \%$, respectively. The blood glucose levels were significantly lower than those of the forskolin treatment group by 48.75 and $46.64 \%$ $(\mathrm{P}<0.01)$. However, there was no significant difference between the diabetic control and forskolin treatment groups (Table I).

Determination of glucose levels in retinal tissues. The retinal glucose levels in the 3 groups were measured. The glucose level in the mouse retina in the normal control group was $(38.21 \pm 2.31 \mathrm{nmol})$ glucose $\cdot \mathrm{mg}$ protein $^{-1}$. At week 21 , the retinal glucose level of the mice in the diabetic control group reached $(152.13 \pm 7.31) \mathrm{nmol}$ glucose $\cdot \mathrm{mg}$ protein ${ }^{-1}$, and the retinal glucose level of the mice in the forskolin treatment group was $(103.54 \pm 6.27)$ nmol glucose $\cdot m g$ protein $^{-1}$. The retinal glucose levels of the mice in the two groups of diabetic models were both higher than that in the normal control group, and the differences were statistically significant $(\mathrm{P}<0.01)$. In addition, the retinal glucose level of the mice in the forskolin treatment group was significantly lower $(\mathrm{P}<0.01)$ than that in the diabetic control group and was approximately $68.06 \%$ of that in the diabetic control group (Fig. 1A).

Retinal GLUT1 expression in the three groups. The expression of GLUT1 in the retina was upregulated under diabetic conditions, but the expression of retinal GLUT1 in the forskolin treatment group was lower than that in the normal control group by $72.13 \%$; however, GLUT1 expression in the forskolin treatment group was lower than that in the diabetic control group by $8.07 \%$. Both of these differences were statistically significant $(\mathrm{P}<0.01)$ (Fig. 1B).

Expression of inflammatory factors in the retina of the mice in the diabetic control group and the forskolin control group. Previous studies confirmed that DR is a chronic inflammatory disease (11). ICAM-1 and TNF- $\alpha$ are two 
A

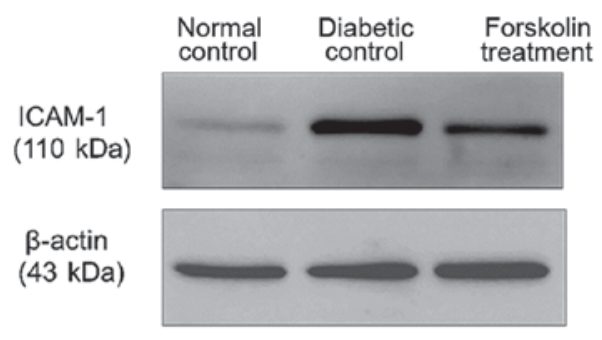

B

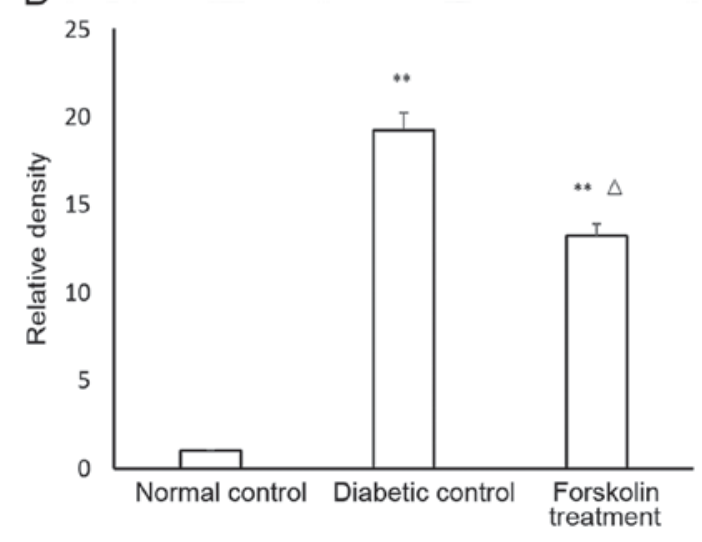

Figure 2. (A) Inflammation factor ICAM-1 expression in retina of three groups mice. (B) Data are representative of three independent experiments and values are expressed in maen $\pm \mathrm{SD}$. ${ }^{* *} \mathrm{P}<0.01$ vs. normal control; ${ }^{\Delta} \mathrm{P}<0.05$ vs. diabetic control, $\mathrm{n}=6$. mean $\pm \mathrm{SD}$.

important markers of inflammatory reactions. The expression of ICAM-1 and TNF- $\alpha$ in the retina of the mice in the three groups was determined using western blot, and the results were statistically analysed. The results showed that the ICAM-1 expression was upregulated in both the diabetic control group and the forskolin treatment group compared to that in the normal control group, and the differences were statistically significant $(\mathrm{P}<0.01)$. In addition, the ICAM-1 expression in the retina of the mice in the forskolin treatment group was approximately $68.75 \%$ of that in the diabetic control group, and the difference was statistically significant $(\mathrm{P}<0.05)$ (Fig. 2).

The TNF- $\alpha$ results were similar. The expression of TNF- $\alpha$ in the diabetic control group and the forskolin treatment group was also upregulated compared to that in the normal control group, with statistically significant differences $(\mathrm{P}<0.01)$. In addition, the TNF- $\alpha$ expression in the retina of the mice in the forskolin treatment group was $\sim 75.37 \%$ of that in the diabetic control group, and the difference was statistically significant $(\mathrm{P}<0.05)$ (Fig. 3).

Leukostasis changes in retinal blood vessels in the diabetic control group and the forskolin treatment group. Leukostasis in retinal blood vessels is an important indicator of retinal inflammation (12) and is also an early pathological change in DR. Our results showed that there were only several isolated adherent leukocytes in the retinal blood vessels in the normal control group. The mice in the diabetic group and the forskolin treatment groups both had more leukostasis; however, the number of counted adherent leukocytes in the forskolin treatment group was approximately $48.28 \%$ of that in the diabetic

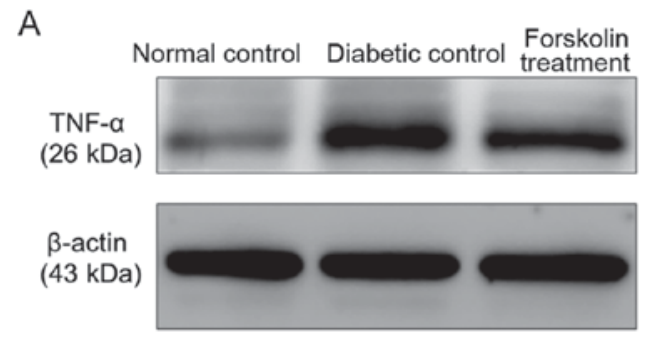

B

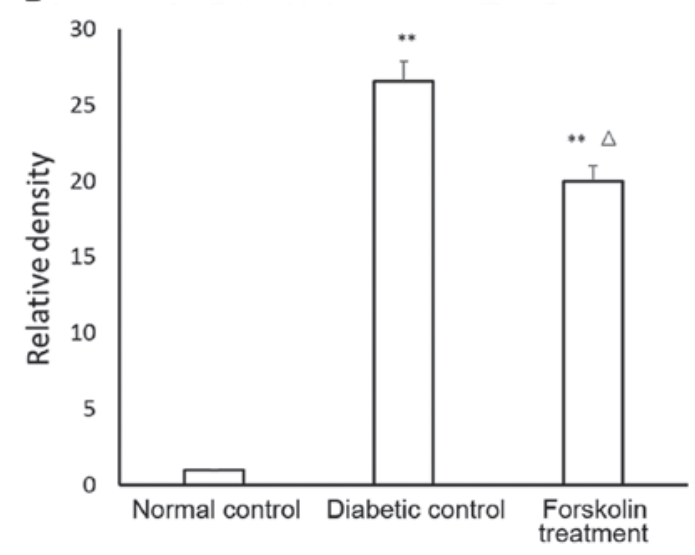

Figure 3. (A) Inflammation factor TNF- $\alpha$ expression in retina of three groups mice. (B) Data are representative of three independent experiments and values are expressed in mean $\pm \mathrm{SD}$. ${ }^{* *} \mathrm{P}<0.01$ vs. normal control; ${ }^{\Delta} \mathrm{P}<0.05$ vs. diabetic control. $n=6$, mean \pm SD.

control group, and the difference was statistically significant $(\mathrm{P}<0.01)$ (Fig. 4).

\section{Discussion}

DR is one of the most common severe complications of DM and will become an extensive public health issue in the future. The specific pathogenic mechanism is still not clear. A large-scale, randomized, clinical study, the UK Prospective Diabetes Study (UKPDS) (13), previously confirmed the important roles of high blood glucose in DR and other diabetic complications. The mechanisms of high glucose on retinal cells may include influences on the expression of specific genes, an increase in advanced glycation end products, and an increase in the oxidative stress reaction (14). We hypothesized that since the high glucose microenvironment of retinal tissues in the diabetic condition exerts damaging effects, controlling the local glucose level in retinal tissues and reversing the high glucose environment may alleviate inflammation response. Glucose in the retina is transported from blood, however, water-soluble glucose cannot pass through the phospholipid bilayer of the cell membrane and requires a group of carrier proteins, GLUTs, for glucose transport. GLUT1 is the only carrier that allows glucose to pass through the blood-retinal barrier (8). Kumagai et al used immunocytochemistry to quantitatively study GLUT1 expression in eyeballs (without or with only mild retinopathy) of DM patients and showed that the GLUT1 activity in the retina of more than half of the eyeballs was 18 times higher than that in the normal control group, suggesting that GLUT1 upregulation mediated cell injury through its 

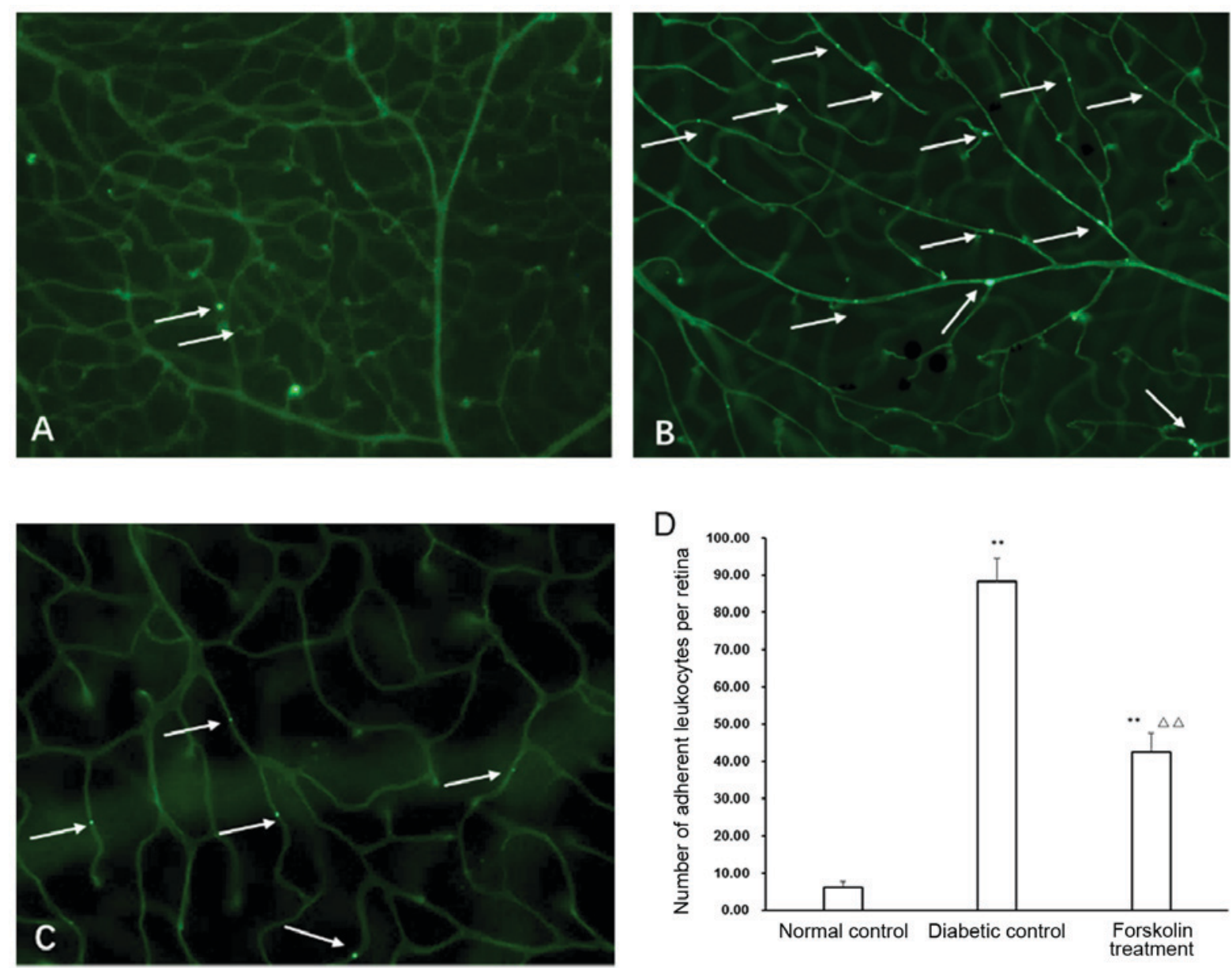

Figure 4. Retinal leukostasis assay of three groups mice. (A) Normal control group; (B) Diabetic control group; (C) forskolin treatment group; and (D) statistical analysis of adherent leukocytes quantification. Data are representative of three independent experiments and values are expressed in mean \pm SD. ${ }^{* * *} \mathrm{P}<0.01$ vs. normal control; ${ }^{\Delta \Delta} \mathrm{P}<0.01$ vs. diabetic control, $\mathrm{n}=6$, mean $\pm \mathrm{SD}$. Fluorescence microscope, original magnification, $\mathrm{x} 400$. White arrows indicate for leukocytes adhere to retinal vasculatures.

transport of a large amount of glucose into the retina (15). Forskolin could competitively inhibit glucose transport by GLUT1, thus reducing retinal glucose levels. As mentioned above, the expression of retinal GLUT1 of the forskolin treatment was decreased by $\sim 95.04 \%$ compared with that in the diabetic control group and by $\sim 72.13 \%$ compared with that in the normal control group. At 21 weeks after the establishment of the DM model, the retinal glucose level of the mice in the forskolin treatment group was only $68.06 \%$ of that in the diabetic control group, confirming that forskolin could effectively limit glucose transport into the retina via GLUT1 inhibition.

Inflammation is an important step in the pathological changes of DR. We measured two markers of the inflammatory reaction, the level of the ICAM-1 chemokine and the level of the TNF- $\alpha$ cytokine. ICAM-1 plays important roles in mediating leukostasis (16). Inhibition of ICAM-1 can significantly reduce leukostasis and vascular permeability (17). TNF- $\alpha$ expression is also upregulated in the retina of DR patients (18). We found that the expression levels of ICAM-1 and TNF- $\alpha$ in the retina of the mice in the forskolin treatment group were only 68.75 and $75.37 \%$, respectively, of those in the diabetic control group. These results suggested that after glucose transport into the retina was inhibited by forskolin, the inflammatory reaction in diabetic mice was relatively mild.

Studies showed that the number of leukocytes in the retina of diabetic animal models increased, the adhesion ability increased, and the deformability decreased $(19,20)$. The passive deformability of leukocytes to pass through capillaries that had smaller diameters in DR patients, thus causing an increase in the leukocyte adhesion rate. In addition, with the progression of the disease, the increase in the adhesion rate was more evident (14). A leukostasis assay in retinal blood vessels could be used to analyse and compare the extent of inflammatory reactions in DR. This study showed that although the forskolin treatment had a higher number of adherent leukocytes in retinal blood vessels than the normal control group, the number was only $48.28 \%$ of that in the diabetic control group.

In conclusion, inhibition of GLUT1 by forskolin could decrease the retinal glucose level in diabetic mice to form a relatively low glucose environment in the retina. Under this environment, retinal inflammation response was relieved compared to that in general diabetic mice. These results suggested that inhibition of GLUT1 to limit local retinal glucose levels may become a new direction for future therapy of DR. 


\section{Acknowledgements}

The present study was supported by the National Natural Science Foundation of China (grant nos. 81460088 and 81760176), Jiangxi Provincial Training Program for Distinguished Young Scholars (grant no. 20171BCB23092), Jiangxi Provincial Key R\&D Program (grant no. 20171BBG70099), Jiangxi Provincial Natural Science Foundation for Youth Scientific Research (grant no. 20171BAB215032), Youth Scientific Research Foundation of the Second Affiliated Hospital of Nanchang University (grant no. 2014YNQN12011) and Traditional Chinese Medicine Scientific Research Program of Jiangxi Health and Family Planning Commission (grant no. 2013B016)

\section{References}

1. International Diabetes Federation (IDF): IDF Diabetes Atlas. 7th edition. IDF, Brussels, 2015.

2. Wong TY,Simó R and Mitchell P: Fenofibrate-a potential systemic treatment for diabetic retinopathy. Am J Ophthalmol 154: 6-12, 2012.

3. Sjølie AK, Dodson P and Hobbs FR: Does renin-angiotensin system blockade have a role in preventing diabetic retinopathy? A clinical review. Int J Clin Pract 65: 148-153, 2011.

4. Chen M, Curtis TM and Stitt AW: Advanced glycation end products and diabetic retinopathy. Curr Med Chem 20: 3234-3240, 2013.

5. Tang $\mathrm{J}$ and Kern TS: Inflammation in diabetic retinopathy. Prog Retin Eye Res 30: 343-358, 2011.

6. Roy S, Kern TS, Song B and Stuebe C: Mechanistic insights into pathological changes in the diabetic retina: Implications for targeting diabetic retinopathy. Am J Pathol 187: 9-19, 2017.

7. Silva PS, Sun JK and Aiello LP: Role of steroids in the management of diabetic macular edema and proliferative diabetic retinopathy. Semin Ophthalmol 24: 93-99, 2009.

8. Thorens B and Mueckler M: Glucose transporters in the 21st century. Am J Physiol Endocrinol Metab 298: E141-E145, 2010.
9. Lu L, Seidel CP, Iwase T, Stevens RK, Gong YY, Wang X, Hackett SF and Campochiaro PA: Suppression of GLUT1; a new strategy to prevent diabetic complications. J Cell Physiol 228: 251-257, 2013.

10. Fu S, Dong S, Zhu M, Sherry DM, Wang C, You Z, Haigh JJ and Le YZ: Müller glia are a major cellular source of survival signals for retinal neurons in diabetes. Diabetes 64: 3554-3563, 2015.

11. Agrawal NK and Kant S: Targeting inflammation in diabetes: Newer therapeutic options. World J Diabetes 5: 697-710, 2014.

12. Wang J, Xu X, Elliott MH, Zhu M and Le YZ: Müller cell-derived VEGF is essential for diabetes-induced retinal inflammation and vascular leakage. Diabetes 59: 2297-2305, 2010.

13. Intensive blood-glucose control with sulphonylureas or insulin compared with conventional treatment and risk of complications in patients with type 2 diabetes (UKPDS 33). UK prospective diabetes study (UKPDS) group. Lancet 352: 837-853, 1998.

14. Stophen J and Ryan R: Retina. 4th edition. Elsevier, Singapore, 2010.

15. Kumagai AK, Vinores SA and Pardridge WM: Pathological upregulation of inner blood-retinal barrier Glutl glucose transporter expression in diabetes mellitus. Brain Res 706: 313-317, 1996.

16. Tian P, Ge H, Liu H, Kern TS, Du L, Guan L, Su S and Liu P: Leukocytes from diabetic patients kill retinal endothelial cells: Effects of berberine. Mol Vis 19: 2092-2105, 2013.

17. Zhang HT, Shi K, Baskota A, Zhou FL, Chen YX and Tian HM: Silybin reduces obliterated retinal capillaries in experimental diabetic retinopathy in rats. Eur J Pharmacol 740: 233-239, 2014.

18. Zhang Q and Steinle JJ: IGFBP-3 inhibits TNF- $\alpha$ production and TNFR-2 signaling to protect against retinal endothelial cell apoptosis. Microvase Res 95: 76-81, 2014.

19. Lutty GA: Effects of diabetes on the eye. Invest Ophthalmol Vis Sci 54: ORSF81-ORSF87, 2013.

20. Noda K, Nakao S, Zandi S, Sun D and Hayes KC, Hafezi-Moghadam A: Retinopathy in a novel model of metabolic syndrome and type 2 diabetes: New insight on the inflammatory paradigm. FASEB J 28: 2038-2046, 2014. 